J o u r n a l of

Mathematics

and Applications

JMA No 37, pp 27-37 (2014)

\title{
On Generalised Quasi-ideals and Bi-ideals in Ternary Semigroups
}

\author{
Manish Kant Dubey, Rohatgi Anuradha
}

\begin{abstract}
In this paper, we introduce the notions of generalised quasi-ideals and generalised bi-ideals in a ternary semigroup. We also characterised these notions in terms of minimal quasi-ideals and minimal bi-ideals in a ternary semigroup.
\end{abstract}

AMS Subject Classification: 16 Y30, 16 Y60

Keywords and Phrases: Ternary Semigroup, Quasi-ideals, Bi-ideals, Minimal Quasiideals, Minimal Bi-ideals

\section{Introduction and Preliminaries}

Good and Hughes[5] introduced the notion of bi-ideals and Steinfeld [2] introduced the notion of quasi-ideals in semigroups. In [1], Sioson studied the concept of quasi-ideals in ternary semigroups. He also introduced the notion of regular ternary semigroups and characterised them by using the notion of quasi-ideals. In [7], Dixit and Dewan studied about the quasi-ideals and bi-ideals of ternary semigroups.

A nonempty set $S$ with a ternary operation $S \times S \times S \longmapsto S$, written as $\left(x_{1}, x_{2}, x_{3}\right) \longmapsto\left[x_{1} x_{2} x_{3}\right]$ is called a ternary semigroup if it satisfies the following associative law: $\left[\left[x_{1} x_{2} x_{3}\right] x_{4} x_{5}\right]=\left[x_{1}\left[x_{2} x_{3} x_{4}\right] x_{5}\right]=\left[x_{1} x_{2}\left[x_{3} x_{4} x_{5}\right]\right]$ for any $x_{1}, x_{2}, x_{3}, x_{4}, x_{5} \in S$. In this paper, we denote $\left[x_{1} x_{2} x_{3}\right]$ by $x_{1} x_{2} x_{3}$.

A non-empty subset $T$ of a ternary semigroup $S$ is called a ternary subsemigroup if $t_{1} t_{2} t_{3} \in T$ for all $t_{1}, t_{2}, t_{3} \in T$. A ternary subsemigroup $I$ of a ternary semigroup $S$ is called a left ideal of $S$ if $S S I \subseteq I$, a lateral ideal if $S I S \subseteq I$, a right ideal of $S$ if $I S S \subseteq I$, a two-sided ideal of $S$ if $I$ is both left and right ideal of $S$, and an ideal of $S$ if $I$ is a left, a right and a lateral ideal of $S$. An ideal $I$ of a ternary semigroup $S$ is called a proper ideal if $I \neq S$. Let $S$ be a ternary semigroup. If there exists an element $0 \in S$ such that $0 x y=x 0 y=x y 0=0$ for all $x, y \in S$, then "0" is called the zero element or simply the zero of the ternary semigroup $S$. In this case $S \cup\{0\}$ becomes a ternary semigroup with zero. For example, the set of all nonpositive integers $Z_{0}^{-1}$ forms a ternary semigroup with usual ternary multiplication

COPYRIGHT (c) by Publishing Department Rzeszów University of Technology P.O. Box 85, 35-959 Rzeszów, Poland 
and ' 0 ' forms a ternary semigroup with zero element and also the zero element satisfy $(S S)^{0} S=S^{0} S S^{0}=S(S S)^{0}=S$. Throughout this paper $S$ will always denote a ternary semigroup with zero. A ternary subsemigroup $Q$ of a ternary semigroup $S$ is called a quasi-ideal of $S$ if $Q S S \cap(S Q S \cup S S Q S S) \cap S S Q \subseteq Q$ and a ternary subsemigroup $B$ of a ternary semigroup $S$ is called a bi-ideal of $S$ if $B S B S B \subseteq B$. It is easy to see that every quasi-ideal in a ternary semigroup is a bi-ideal of $S$. An element $a$ in a ternary semigroup $S$ is called regular if there exists an element $x$ in $S$ such that $a x a=a$. A ternary semigroup is called regular if all of its elements are regular. A ternary semigroup $S$ is regular if and only if $R \cap M \cap L=R M L$ for every right ideal $R$, lateral ideal $M$ and left ideal $L$ of $S$.

\section{Generalised Quasi-ideals in Ternary Semigroup}

In this section, we introduce the concept of generalised quasi-ideals in ternary semigroups and prove some results related to the same.

Definition 2.1. A ternary subsemigroup $Q$ of a ternary semigroup $S$ is called a generalised quasi-ideal or $(m,(p, q), n)$-quasi-ideal of $S$ if $Q(S S)^{m} \cap\left(S^{p} Q S^{q} \cup S^{p} S Q S S^{q}\right) \cap$ $(S S)^{n} Q \subseteq Q$, where $m, n, p, q$ are positive integers greater than 0 and $p+q=$ even.

Remark 2.1. Every quasi-ideal of a ternary semigroup $S$ is $(1,(1,1), 1)$-quasi-ideal of $S$. But $(m,(p, q), n)$-quasi-ideal of a ternary semigroup $S$ need not be a quasi-ideal of $S$.

Example 1. Let $Z^{-} \backslash\{-1\}$ be the set of all negative integers excluding $\{0\}$. Then $Z^{-} \backslash\{-1\}$ is a ternary semigroup with usual ternary multiplication. Consider $Q=$ $\{-3\} \cup\left\{k \in Z^{-}: k \leq-14\right\}$. Clearly $Q$ is a non-empty ternary subsemigroup of $S$ and also $Q$ is $(2,(1,1), 3)$-quasi-ideal of $S$. Now, $\{-12\} \in Q S S \cap(S Q S \cup S S Q S S) \cap S S Q$. But $\{-12\} \notin Q$. Therefore $Q S S \cap(S Q S \cup S S Q S S) \cap S S Q \nsubseteq Q$. Hence $Q$ is not quasi-ideal of $Z^{-} \backslash\{-1\}$.

Lemma 2.1. Non-empty intersection of arbitrary collection of ternary subsemigroups of a ternary semigroup $S$ is a ternary subsemigroup of $S$.

Proof. Let $T_{i}$ be a ternary subsemigroup of $S$ for all $i \in I$ such that $\bigcap_{i \in I} T_{i} \neq \emptyset$. Let $t_{1}, t_{2}, t_{3} \in \bigcap_{i \in I} T_{i}$. Then $t_{1}, t_{2}, t_{3} \in T_{i}$ for all $i \in I$. Since $T_{i}$ is a ternary subsemigroup of $S$ for all $i \in I$, therefore $t_{1} t_{2} t_{3} \in T_{i}$ for all $i \in I$. Therefore $t_{1} t_{2} t_{3} \in \bigcap_{i \in I} T_{i}$. Hence $\bigcap_{i \in I} T_{i}$ is a ternary subsemigroup of $S$.

Theorem 2.1. Let $S$ be a ternary semigroup and $Q_{i}$ be an $(m,(p, q), n)$-quasi-ideal of $S$ such that $\bigcap_{i \in I} Q_{i} \neq \emptyset$. Then $\bigcap_{i \in I} Q_{i}$ is an $(m,(p, q), n)$-quasi-ideal of $S$.

Proof. Clearly $\bigcap_{i \in I} Q_{i}$ is a ternary subsemigroup of $S$ (by Lemma 2.1).

Let $x \in\left[\bigcap_{i \in I} Q_{i}(S S)^{m}\right] \cap\left[S^{p} \bigcap_{i \in I} Q_{i} S^{q} \cup S^{p} S \bigcap_{i \in I} Q_{i} S S^{q}\right] \cap\left[(S S)^{n} \bigcap_{i \in I} Q_{i}\right]$. Then $x \in$ 
$\bigcap_{i \in I} Q_{i}(S S)^{m}, x \in S^{p} \bigcap_{i \in I} Q_{i} S^{q} \cup S^{p} S \bigcap_{i \in I} Q_{i} S S^{q}$ and $x \in(S S)^{n} \bigcap_{i \in I} Q_{i}$. This implies $x \in Q_{i}(S S)^{m}, x \in\left[S^{p} Q_{i} S^{q} \cup S^{p} S Q_{i} S S^{q}\right]$ and $x \in(S S)^{n} Q_{i}$ for all $i \in I$. Therefore $x \in\left[Q_{i}(S S)^{m}\right] \cap\left[S^{p} Q_{i} S^{q} \cup S^{p} S Q_{i} S S^{q}\right] \cap\left[(S S)^{n} Q_{i}\right] \subseteq Q_{i}$ for all $i \in I$, since $Q_{i}$ is an $(m,(p, q), n)$-quasi-ideal of $S$. Thus $x \in Q_{i}$ for all $i \in I$. Therefore $x \in \bigcap_{i \in I} Q_{i}$. Hence $\bigcap_{i \in I} Q_{i}$ is an $(m,(p, q), n)$-quasi-ideal of $S$.

Remark 2.2. Let $Z^{-}$be the set of all negative integers under ternary multiplication and $Q_{i}=\left\{k \in Z^{-}: k \leq-i\right\}$ for all $i \in I$. Then $Q_{i}$ is an $(2,(1,1), 3)$-quasi-ideal of $Z^{-}$for all $i \in I$. But $\bigcap_{i \in I} Q_{i}=\emptyset$. So condition $\bigcap_{i \in I} Q_{i} \neq \emptyset$ is necessary.

Definition 2.2. Let $S$ be a ternary semigroup. Then a ternary subsemigroup

(i) $R$ of $S$ is called an $m$-right ideal of $S$ if $R(S S)^{m} \subseteq R$.

(ii) $M$ of $S$ is called an $(p, q)$-lateral ideal of $S$ if $S^{p} M S^{q} \cup S^{p} S M S S^{q} \subseteq M$,

(iii) $L$ of $S$ is called an n-left ideal of $S$ if $(S S)^{n} L \subseteq L$,

where $m, n, p, q$ are positive integers and $p+q$ is an even positive integer.

Theorem 2.2. Every m-right, $(p, q)$-lateral and n-left ideal of a ternary semigroup $S$ is an $(m,(p, q), n)$-quasi-ideal of $S$. But converse need not be true.

Proof. One way is straight forward. Conversely, let $S=M_{2}\left(Z_{0}^{-}\right)$be the ternary semigroup of $2 \times 2$ square matrices over $Z_{0}^{-}$. Consider $Q=\left\{\left(\begin{array}{ll}a & 0 \\ 0 & 0\end{array}\right): a \in Z_{0}^{-}\right\}$. Then $Q$ is an $(2,(1,1), 3)$-quasi-ideal of $S$. But it is not 2-right ideal, (1,1)-lateral ideal and 3-left ideal of $S$.

Theorem 2.3. Let $S$ be a ternary semigroup. Then the following statements hold:

(i) Let $R_{i}$ be an m-right ideal of $S$ such that $\bigcap_{i \in I} R_{i} \neq \emptyset$. Then $\bigcap_{i \in I} R_{i}$ is an m-right ideal of $S$.

(ii) Let $M_{i}$ be an $(p, q)$-lateral ideal of $S$ such that $\bigcap_{i \in I} M_{i} \neq \emptyset$. Then $\bigcap_{i \in I} M_{i}$ is an $(p, q)$-lateral ideal of $S$.

(iii) Let $L_{i}$ be an $n$-left ideal of $S$ such that $\bigcap_{i \in I} L_{i} \neq \emptyset$. Then $\bigcap_{i \in I} L_{i}$ is an $n$-left ideal of $S$.

Proof. Similar to the proof of Theorem 2.1

Theorem 2.4. Let $R$ be an $m$-right ideal, $M$ be an $(p, q)$-lateral ideal and $L$ be an $n$-left ideal of a ternary semigroup $S$. Then $R \cap M \cap L$ is an $(m,(p, q), n)$-quasi-ideal of $S$.

Proof. Suppose $Q=R \cap M \cap L$. Since every $m$-right, $(p, q)$-lateral and $n$-left ideal of ternary semigroup $S$ is an $(m,(p, q), n)$-quasi-ideal of $S$, therefore $R, M$ and $L$ are $(m,(p, q), n)$-quasi-ideals of $S$. Clearly, $R \cap M \cap L$ is non-empty. By Theorem 2.1, we have $Q=R \cap M \cap L$ is an $(m,(p, q), n)$-quasi-ideal of $S$. 
Lemma 2.2. Let $Q$ be an $(m,(p, q), n)$-quasi-ideal of a ternary semigroup $S$. Then

(i) $R=Q \cup Q(S S)^{m}$ is an m-right ideal of $S$.

(ii) $M=Q \cup\left(S^{p} Q S^{q} \cup S^{p} S Q S S^{q}\right)$ is an $(p, q)$-lateral ideal of $S$.

(iii) $L=Q \cup(S S)^{n} Q$ is an n-left ideal of $S$.

Proof. It is easy to show that $R$ is ternary subsemigroup of $S$. Now to show that $R$ is an $m$-right ideal of $S$.

$$
\begin{aligned}
R(S S)^{m} & =\left[\left(Q \cup Q(S S)^{m}\right](S S)^{m}\right. \\
& =Q(S S)^{m} \cup Q(S S)^{m}(S S)^{m} \\
& =Q(S S)^{m} \cup Q(S S S S)^{m} \\
& \subseteq Q(S S)^{m} \cup Q(S S)^{m} \\
& =Q(S S)^{m} \subseteq R .
\end{aligned}
$$

Therefore $R$ is an $m$-right ideal of $S$. Similarly, we can show that $M$ is an $(p, q)$-lateral ideal of $S$ and $L$ is an $n$-left ideal of $S$.

Theorem 2.5. Every $(m,(p, q), n)$-quasi-ideal in a regular ternary semigroup $S$ is the intersection of $m$-right, $(p, q)$-lateral and $n$-left ideal of $S$.

Proof. Let $S$ be regular ternary semigroup and $Q$ be an $(m,(p, q), n)$-quasi-ideal of $S$. Then $R=Q \cup Q(S S)^{m}, M=Q \cup\left(S^{p} Q S^{q} \cup S^{p} S Q S S^{q}\right)$ and $L=Q \cup(S S)^{n} Q$ are $m$-right, $(p, q)$ - lateral and $n$-left ideal of $S$ respectively. Clearly $Q \subseteq R, Q \subseteq M$ and $Q \subseteq L$ implies $Q \subseteq R \cap M \cap L$. Since $S$ is regular therefore $Q \subseteq Q(S S)^{m}$, $Q \subseteq S^{p} Q S^{q} \cup S^{p} S Q S S^{q}$ and $Q \subseteq(S S)^{n} Q$.

Thus $R=Q(S S)^{m}, M=S^{p} Q S^{q} \cup S^{p} S Q S S^{q}$ and $L=(S S)^{n} Q$. Now

$$
R \cap M \cap L=Q(S S)^{m} \cap\left(S^{p} Q S^{q} \cup S^{p} S Q S S^{q}\right) \cap(S S)^{n} Q \subseteq Q
$$

Hence, $Q=R \cap M \cap L$.

\section{Generalised Minimal Quasi-ideals}

In this section, we study the concept of generalised minimal quasi-ideal or minimal $(m,(p, q), n)$-quasi-ideals of ternary semigroup $S$.

An $(m,(p, q), n)$-quasi-ideal $Q$ of a ternary semigroup $S$ is called minimal $(m,(p, q), n)$-quasi-ideal of $S$ if $Q$ does not properly contain any $(m,(p, q), n)$-quasiideal of $S$. Similarly, we can define minimal $m$-right ideals, minimal $(p, q)$-lateral ideals and minimal $n$-left ideals of a ternary semigroup.

Lemma 3.1. Let $S$ be a ternary semigroup and $a \in S$. Then the following statements hold:

(i) $a(S S)^{m}$ is an m-right ideal of $S$.

(ii) $\left(S^{p} a S^{q} \cup S^{p} S a S S^{q}\right)$ is an $(p, q)$-lateral ideal of $S$. 
(iii) $(S S)^{n} a$ is an n-left ideal of $S$.

(iv) $a(S S)^{m} \cap\left(S^{p} a S^{q} \cup S^{p} S a S S^{q}\right) \cap(S S)^{n} a$ is an $(m,(p, q), n)$-quasi-ideal of $S$.

Proof. (i), (ii) and (iii) are obvious. (iv) follows from (i), (ii), (iii) and Theorem 2.4.

Theorem 3.1. Let $S$ be a ternary semigroup and $Q$ be an $(m,(p, q), n)$-quasi-ideal of $S$. Then $Q$ is minimal iff $Q$ is the intersection of some minimal m-right ideal $R$, minimal $(p, q)$-lateral ideal $M$ and minimal $n$-left ideal $L$ of $S$.

Proof. Suppose $Q$ is minimal $(m,(p, q), n)$-quasi-ideal of $S$. Let $a \in Q$. Then by above Lemma, we have $a(S S)^{m}$ is an $m$-right ideal, $\left(S^{p} a S^{q} \cup S^{p} S a S S^{q}\right)$ is an $(p, q)$-lateral ideal, $(S S)^{n} a$ is an $n$-left ideal and $a(S S)^{m} \cap\left(S^{p} a S^{q} \cup S^{p} S a S S^{q}\right) \cap(S S)^{n} a$ is an $(m,(p, q), n)$-quasi-ideal of $S$. Now,

$$
\begin{aligned}
a(S S)^{m} & \cap\left(S^{p} a S^{q} \cup S^{p} S a S S^{q}\right) \cap(S S)^{n} a \\
& \subseteq Q(S S)^{m} \cap\left(S^{p} Q S^{q} \cup S^{p} S Q S S^{q}\right) \cap(S S)^{n} Q \\
& \subseteq Q .
\end{aligned}
$$

Since $Q$ is minimal therefore $a(S S)^{m} \cap\left(S^{p} a S^{q} \cup S^{p} S a S S^{q}\right) \cap(S S)^{n} a=Q$.

Now, to show that $a(S S)^{m}$ is minimal $m$-right ideal of $S$. Let $R$ be an $m$-right ideal of $S$ contained in $a(S S)^{m}$. Then

$$
\begin{aligned}
R & \cap\left(S^{p} a S^{q} \cup S^{p} S a S S^{q}\right) \cap(S S)^{n} a \\
& \subseteq a(S S)^{m} \cap\left(S^{p} a S^{q} \cup S^{p} S a S S^{q}\right) \cap(S S)^{n} a \\
& =Q .
\end{aligned}
$$

Since $R \cap\left(S^{p} a S^{q} \cup S^{p} S a S S^{q}\right) \cap(S S)^{n} a$ is an $(m,(p, q), n)$-quasi-ideal of $S$ and $Q$ is minimal, therefore $R \cap\left(S^{p} a S^{q} \cup S^{p} S a S S^{q}\right) \cap(S S)^{n} a=Q$. This implies $Q \subseteq R$ and therefore

$$
a(S S)^{m} \subseteq Q(S S)^{m} \subseteq R(S S)^{m} \subseteq R
$$

implies $R=a(S S)^{m}$. Thus $m$-right ideal $a(S S)^{m}$ is minimal. Similarly, we can prove that $\left(S^{p} a S^{q} \cup S^{p} S a S S^{q}\right)$ is minimal $(p, q)$-lateral ideal of $S$ and $(S S)^{n} a$ is minimal $n$-left ideal of $S$.

Conversely, assume that $Q=R \cap M \cap L$ for some minimal $m$-right ideal $R$, minimal $(p, q)$-lateral ideal $M$ and minimal $n$-left ideal $L$. So, $Q \subseteq R, Q \subseteq M$ and $Q \subseteq L$. Let $Q^{\prime}$ be an $(m,(p, q), n)$-quasi-ideal of $S$ contained in $Q$. Then $Q^{\prime}(S S)^{m} \subseteq Q(S S)^{m} \subseteq$ $R(S S)^{m} \subseteq R$. Similarly, $\left(S^{p} Q^{\prime} S^{q} \cup S^{p} S Q^{\prime} S S^{q}\right) \subseteq M$ and $(S S)^{n} Q^{\prime} \subseteq(S S)^{n} Q \subseteq L$.

Now $Q^{\prime}(S S)^{m}$ is an $m$-right ideal of $S$, as $Q^{\prime}(S S)^{m}(S S)^{m} \subseteq Q^{\prime}(S S)^{m}$. Similarly, $\left(S^{p} Q^{\prime} S^{q} \cup S^{p} S Q^{\prime} S S^{q}\right)$ is an $(p, q)$-lateral ideal of $S$ and $(S S)^{n} Q^{\prime}$ is an $n$-left ideal of $S$. Since $R, M$ and $L$ are minimal $m$-right ideal, minimal $(p, q)$-lateral ideal and minimal $n$-left ideal of $S$ respectively, therefore $Q^{\prime}(S S)^{m}=R, S^{p} Q^{\prime} S^{q} \cup S^{p} S Q^{\prime} S S^{q}=M$ and $(S S)^{n} Q^{\prime}=L$.

Thus $Q=R \cap M \cap L=Q^{\prime}(S S)^{m} \cap\left(S^{p} Q^{\prime} S^{q} \cup S^{p} S Q^{\prime} S S^{q}\right) \cap(S S)^{n} Q^{\prime} \subseteq Q^{\prime}$. Hence $Q=Q^{\prime}$. Thus $Q$ is minimal $(m,(p, q), n)$-quasi-ideal of $S$. 
Note. A ternary semigroup $S$ need not contains a minimal $(m,(p, q), n)$-quasi-ideal of $S$.

For example, let $Z^{-}$be the set of all negative integers. Then $Z^{-}$is a ternary semigroup with usual ternary multiplication. Let $Q=\{-2,-3,-4, \ldots\}$. Then $Q$ is an $(2,(1,1), 3)$-quasi-ideal of $Z^{-}$. Suppose $Q$ is minimal $(2,(1,1), 3)$-quasi-ideal of $Z^{-}$. Let $Q^{\prime}=Q \backslash\{-2\}$. Then we can easily show that $Q^{\prime}$ is an $(2,(1,1), 3)$-quasi-ideal of $Z^{-}$. But $Q^{\prime}$ is proper subset of $Q$. This is contradiction. Hence, $Z^{-}$does not contain a minimal $(m,(p, q), n)$-quasi-ideal.

Theorem 3.2. Let $S$ be a ternary semigroup. Then the following holds:

(i) An m-right ideal $R$ is minimal iff $a(S S)^{m}=R$ for all $a \in R$.

(ii) An $(p, q)$-lateral ideal $M$ is minimal iff $\left(S^{p} a S^{q} \cup S^{p} S a S S^{q}\right)=M$ for all $a \in M$.

(iii) An n-left ideal $L$ is minimal iff $(S S)^{n} a=L$ for all $a \in L$.

(iv) $A n(m,(p, q), n)$-quasi-ideal $Q$ is minimal iff $a(S S)^{m} \cap\left(S^{p} a S^{q} \cup S^{p} S a S S^{q}\right) \cap$ $(S S)^{n} a=Q$ for all $a \in Q$.

Proof. (i) Suppose $m$-right ideal $R$ is minimal. Let $a \in R$. Then $a(S S)^{m} \subseteq R(S S)^{m} \subseteq$ $R$. By Lemma 3.1, we have $a(S S)^{m}$ is an $m$-right ideal of $S$. Since $R$ is minimal $m$ right ideal of $S$ therefore $a(S S)^{m}=R$.

Conversely, Suppose that $a(S S)^{m}=R$ for all $a \in R$. Let $R^{\prime}$ be an $m$-right ideal of $S$ contained in $R$. Let $x \in R^{\prime}$. Then $x \in R$. By assumption, we have $x(S S)^{m}=R$ for all $x \in R . R=x(S S)^{m} \subseteq R^{\prime}(S S)^{m} \subseteq R^{\prime}$. This implies $R \subseteq R^{\prime}$. Thus, $R=R^{\prime}$. Hence, $R$ is minimal $m$-right ideal.

Similarly we can prove (ii), (iii) and (iv).

\section{Generalised Bi-ideals in Ternary Semigroup}

In this section, we define generalised bi-ideals in a ternary semigroup and give their characterizations.

Definition 4.1. A ternary subsemigroup $B$ of a ternary semigroup $S$ is called a generalised bi-ideal or $(m,(p, q), n)$ bi-ideal of $S$ if $B(S S)^{m-1} S^{p} B S^{q}(S S)^{n-1} B \subseteq B$, where $m, n, p, q$ are positive integers greater than zero and $p$ and $q$ are odd.

Remark. Every bi-ideal of a ternary semigroup $S$ is $(1,(1,1), 1)$-bi-ideal of $S$. But every $(m,(p, q), n)$-bi-ideal of a ternary semigroup $S$ need not be a bi-ideal of $S$ which is illustrated by the following example.

Example 2. Let $Z^{-} \backslash\{-1\}$ be the set of all negative integers excluding $\{0\}$. Then $Z^{-} \backslash\{-1\}$ is a ternary semigroup with usual ternary multiplication. Consider $B=$ $\{-3,-27\} \cup\left\{k \in Z^{-}: k \leq-110\right\}$. Clearly $B$ is a non-empty ternary subsemigroup of $S$ and also $B$ is $(3,(1,1), 4)$-bi-ideal of $S$. Now $-108 \in B S B S B$. But $-108 \notin B$. Therefore $B S B S B \nsubseteq B$. Hence $B$ is not a bi-ideal of $Z^{-} \backslash\{-1\}$.

Theorem 4.1. Let $S$ be a ternary semigroup and $B_{i}$ be an $(m,(p, q), n)$-bi-ideals of $S$ such that $\bigcap_{i \in I} B_{i} \neq \emptyset$. Then $\bigcap_{i \in I} B_{i}$ is an $(m,(p, q), n)$ bi-ideal of $S$. 
Proof. It is straight forward.

Remark. Let $Z^{-}$be the set of all negative integers. Then $Z^{-}$is a ternary semigroup under usual ternary multiplication and $B_{i}=\left\{k \in Z^{-}: k \leq-i\right\}$ for all $i \in I$. Then $B_{i}$ is an $(3,(1,1), 4)$-bi-ideal of $Z^{-}$for all $i \in I$. But $\bigcap_{i \in I} B_{i}=\emptyset$. So condition $\bigcap_{i \in I} B_{i} \neq \emptyset$ is necessary.

Theorem 4.2. Every $(m,(p, q), n)$-quasi-ideal of a ternary semigroup $S$ is an $(m,(p, q), n)$-bi-ideal of $S$.

Proof. Let $Q$ be an $(m,(p, q), n)$-quasi-ideal of $S$. Then

$$
Q(S S)^{m-1} S^{p} Q S^{q}(S S)^{n-1} Q \subseteq Q(S S)^{m-1} S^{p} S S^{q}(S S)^{n-1} S \subseteq Q(S S)^{m} .
$$

Similarly,

$$
Q(S S)^{m-1} S^{p} Q S^{q}(S S)^{n-1} Q \subseteq S(S S)^{m-1}\left(S^{p} Q S^{q}\right)(S S)^{n-1} S \subseteq S^{p+1} Q S^{q+1} .
$$

Again $\{0\} \subseteq S^{p} Q S^{q}$. So

$$
Q(S S)^{m-1} S^{p} Q S^{q}(S S)^{n-1} Q \subseteq S^{p} Q S^{q} \cup S^{p+1} Q S^{q+1} .
$$

Also,

$$
Q(S S)^{m-1} S^{p} Q S^{q}(S S)^{n-1} Q \subseteq S(S S)^{m-1} S^{p} S S^{q}(S S)^{n-1} Q \subseteq(S S)^{n} Q .
$$

Consequently,

$Q(S S)^{m-1} S^{p} Q S^{q}(S S)^{n-1} Q \subseteq Q(S S)^{m} \cap\left(S^{p} Q S^{q} \cup S^{p+1} Q S^{q+1}\right) \cap(S S)^{n} Q \subseteq Q$.

Hence $Q$ is an $(m,(p, q), n)$-bi-ideal of $S$.

Remark. Every $(m,(p, q), n)$-bi-ideal need not be an $(m,(p, q), n)$-quasi-ideal of $S$ which is illustrated by the following example.

Example 3. Consider the ternary semigroup $S=Z^{-} \backslash\{-1\}$ with usual ternary multiplication and let $B=\{-3,-27\} \cup\left\{k \in Z^{-}: k \leq-194\right\}$. Clearly, $B$ is non-empty ternary subsemigroup of $S$ and also $B$ is $(2,(1,1), 3)$-bi-ideal of $S$. Now, $-192 \in B(S S)^{2} \cap(S B S \cup S S B S S) \cap(S S)^{3} B$. But $-192 \notin B$. Therefore $B(S S)^{2} \cap(S B S \cup S S B S S) \cap(S S)^{3} B \nsubseteq B$. Hence $B$ is not $(2,(1,1), 3)$-quasi-ideal of $S$.

Theorem 4.3. A ternary subsemigroup $B$ of a regular ternary semigroup $S$ is an $(m,(p, q), n)$-bi-ideal of $S$ if and only if $B=B S B$.

Proof. Suppose $B$ is an $(m,(p, q), n)$-bi-ideal of a regular ternary semigroup $S$. Let $b \in B$. Then there exists $x \in S$ such that $b=b x b$. This implies that $b \in B S B$. Hence $B \subseteq B S B$. Now,

$$
B S B \subseteq B S B S B S B S B \subseteq B(S S)(S B S)(S S) B \subseteq B .
$$


Therefore $B=B S B$.

Conversely, if $B=B S B$, then

$$
B(S S)^{m-1} S^{p} B S^{q}(S S)^{n-1} B \subseteq B(S S)^{m-1} S^{p} S S^{q}(S S)^{n-1} B \subseteq B S B=B .
$$

Hence $B$ is an $(m,(p, q), n)$-bi-ideal of $S$.

Theorem 4.4. Let $S$ be a regular ternary semigroup. Then every $(m,(p, q), n)$-biideal of $S$ is an $(m,(p, q), n)$-quasi-ideal of $S$.

Proof. Let $B$ be an $(m,(p, q), n)$-bi-ideal of $S$. Let $a \in B(S S)^{m} \cap\left(S^{p} B S^{q} \cup\right.$ $\left.S^{p} S B S S^{q}\right) \cap(S S)^{n} B$. Then $a \in B(S S)^{m}, a \in\left(S^{p} B S^{q} \cup S^{p} S B S S^{q}\right)$ and $a \in(S S)^{n} B$. Thus $a=b(S S)^{m}=S^{p} b^{\prime} S^{q} \cup S^{p} S b^{\prime \prime} S S^{q}=(S S)^{n} b^{\prime \prime \prime}$ for some $b, b^{\prime}, b^{\prime \prime}, b^{\prime \prime \prime} \in B$. Since $S$ is regular, therefore for $a \in S$ there exists an element $x$ in $S$ such that $a=a x a$. Then

$$
\begin{aligned}
a=a x a & =\text { axaxa } \\
& =b(S S)^{m} x\left(S^{p} b^{\prime} S^{q} \cup S^{p} S b^{\prime \prime} S S^{q}\right) x(S S)^{n} b^{\prime \prime \prime} \\
& \in B(S S)^{m} S\left(S^{p} B S^{q} \cup S^{p} S B S S^{q}\right) S(S S)^{n} B \\
& =\left[B(S S)^{m} S S^{p} B S^{q} S(S S)^{n} B\right] \cup\left[B(S S)^{m} S S^{p} S B S S^{q} S(S S)^{n} B\right] \\
& \subseteq B\left[(S S)^{m} S S^{p} S S^{q} S(S S)^{n}\right] B \cup B\left[(S S)^{m} S S^{p} S S S S^{q} S(S S)^{n}\right] B \\
& \subseteq B S B \cup B S B=B \cup B=B .
\end{aligned}
$$

Thus $a \in B$. Therefore $B(S S)^{m} \cap\left(S^{p} B S^{q} \cup S^{p} S B S S^{q}\right) \cap(S S)^{n} B \subseteq B$. Hence $B$ is an $(m,(p, q), n)$-quasi-ideal of $S$.

It is easy to prove the following propositions:

Proposition 4.5. The intersection of an $(m,(p, q), n)$-bi-ideal $B$ of a ternary semigroup $S$ with a ternary subsemigroup $T$ of $S$ is either empty or an $(m,(p, q), n)$-bi-ideal of $T$.

Proposition 4.6. Let $B$ be an $(m,(p, q), n)$-bi-ideal of a ternary semigroup $S$ and $T_{1}, T_{2}$ are two ternary subsemigroups of $S$. Then $B T_{1} T_{2}, T_{1} B T_{2}$ and $T_{1} T_{2} B$ are $(m,(p, q), n)$-bi-ideals of $S$.

Proposition 4.7. Let $B_{1}, B_{2}$ and $B_{3}$ are three $(m,(p, q), n)$-bi-ideals of a ternary semigroup $S$. Then $B_{1} B_{2} B_{3}$ is an $(m,(p, q), n)$-bi-ideal of $S$.

Proposition 4.8. Let $Q_{1}, Q_{2}$ and $Q_{3}$ are three $(m,(p, q), n)$-quasi-ideals of a ternary semigroup $S$. Then $Q_{1} Q_{2} Q_{3}$ is an $(m,(p, q), n)$-bi-ideal of $S$.

Proposition 4.9. Let $R$ be an m-right, $M$ be an $(p, q)$-lateral and $L$ be an n-left ideal of a ternary semigroup $S$. Then the ternary subsemigroup $B=R M L$ of $S$ is an $(m,(p, q), n)$-bi-ideal of $S$.

Theorem 4.10. Let $S$ be a regular ternary semigroup. If $B$ is an $(m,(p, q), n)$-bi-ideal of $S$, then $B(S S)^{m-1} S^{p} B S^{q}(S S)^{n-1} B=B$. 
Proof. Let $B$ be an $(m,(p, q), n)$-bi-ideal of $S$. Let $a \in B$. Then $a \in S$. Since $S$ is regular, therefore there exists $x \in S$ such that $a=a x a$. Now $a=a x a=a(x a)(x a x)(a x) a \in$ $B(S S)(S B S)(S S) B$. Similarly, by property of regularity it is easy to show that $a \in B(S S)^{m-1} S^{p} B S^{q}(S S)^{n-1} B$. Thus, $B \subseteq B(S S)^{m-1} S^{p} B S^{q}(S S)^{n-1} B$. Since $B$ is an $(m,(p, q), n)$-bi-ideal of $S$, therefore $B(S S)^{m-1} S^{p} B S^{q}(S S)^{n-1} B \subseteq B$. Hence $B(S S)^{m-1} S^{p} B S^{q}(S S)^{n-1} B=B$

Corollary 4.1. Let $S$ be a regular ternary semigroup. If $Q$ is an $(m,(p, q), n)$-quasiideal of $S$, then $Q(S S)^{m-1} S^{p} Q S^{q}(S S)^{n-1} Q=Q$.

Proof. Since every $(m,(p, q), n)$-quasi-ideal of $S$ is an $(m,(p, q), n)$-bi-ideal of $S$, therefore result follows directly.

\section{Generalised Minimal Bi-ideals}

In this section, we introduce the concept of generalised minimal bi-ideal or minimal $(m,(p, q), n)$-bi-ideals in ternary semigroups.

Definition 5.1. An $(m,(p, q), n)$-bi-ideal $B$ of a ternary semigroup $S$ is called minimal $(m,(p, q), n)$-bi-ideal of $S$ if $B$ does not properly contain any $(m,(p, q), n)$-bi-ideal of $S$.

Lemma 5.1. Let $S$ be a ternary semigroup and $a \in S$. Then the following holds:

(i) $a(S S)^{m-1}$ is an $m$-right ideal of $S$.

(ii) $S^{p} a S^{q}$ is an $(p, q)$-lateral ideal of $S$.

(iii) $(S S)^{n-1} a$ is an $n$-left ideal of $S$.

(iv) $a(S S)^{m-1} S^{p} a S^{q}(S S)^{n-1} a$ is an $(m,(p, q), n)$-bi-ideal.

Proof. (i), (ii) and (iii) are obvious and (iv) follows from (i), (ii), (iii).

Theorem 5.1. Let $S$ be a ternary semigroup and $B$ be an $(m,(p, q), n)$-bi-ideal of $S$. Then $B$ is minimal if and only if $B$ is the product of some minimal m-right ideal $R$, minimal $(p, q)$-lateral ideal $M$ and minimal $n$-left ideal $L$ of $S$.

Proof. Suppose $B$ is minimal $(m,(p, q), n)$-bi-ideal of $S$. Let $a \in B$. Then by above Lemma, $a(S S)^{m-1}$ is an $m$-right ideal, $S^{p} a S^{q}$ is an $(p, q)$-lateral ideal, $(S S)^{n-1} a$ is an $n$-left ideal and $a(S S)^{m-1} S^{p} a S^{q}(S S)^{n-1} a$ is an $(m,(p, q), n)$-bi-ideal of $S$. Now $a(S S)^{m-1} S^{p} a S^{q}(S S)^{n-1} a \subseteq B(S S)^{m-1} S^{p} B S^{q}(S S)^{n-1} B \subseteq B$. Since $B$ is minimal, therefore $a(S S)^{m-1} S^{p} a S^{q}(S S)^{n-1} a=B$. Now to show that $a(S S)^{m-1}$ is minimal $m$-right ideal of $S$. Let $R$ be an $m$-right ideal of $S$ contained in $a(S S)^{m-1}$. Then $R\left(S^{p} a S^{q}\right)(S S)^{n-1} a \subseteq a(S S)^{m-1}\left(S^{p} a S^{q}\right)(S S)^{n-1} a=B$. Since $R S^{p} a S^{q}(S S)^{n-1} a$ is an $(m,(p, q), n)$-bi-ideal of $S$ and $B$ is minimal, therefore $R\left(S^{p} a S^{q}\right)(S S)^{n-1} a=B$. This implies $B \subseteq R$. Therefore $a(S S)^{m-1} \subseteq B(S S)^{m-1} \subseteq R(S S)^{m-1} \subseteq R$. Thus $a(S S)^{m-1}$ is minimal. Similarly we can prove that $S^{p} a S^{q}$ is minimal $(p, q)$-lateral ideal of $S$ and $(S S)^{n-1} a$ is minimal $n$-left ideal of $S$. 
Conversely, assume that $B=R M L$ for some minimal $m$-right ideal $R$, minimal $(p, q)$-lateral ideal $M$ and minimal $n$-left ideal $L$. So $B \subseteq R, B \subseteq M$ and $B \subseteq L$. Let $B^{\prime}$ be an $(m,(p, q), n)$-bi-ideal of $S$ contained in $B$. Then $B^{\prime}(S S)^{m-1} \subseteq B(S S)^{m-1} \subseteq$ $R(S S)^{m-1} \subseteq R$. Similarly, $S^{p} B^{\prime} S^{q} \subseteq S^{p} B S^{q} \subseteq S^{p} M S^{q} \subseteq M$ and $(S S)^{n-1} B^{\prime} \subseteq$ $(S S)^{n-1} B \subseteq(S S)^{n-1} L \subseteq L$. Now, $B^{\prime}(S S)^{m-1}(S S)^{m} \subseteq B^{\prime}(S S)^{m-1}$. So $B^{\prime}(S S)^{m-1}$ is an $m$-right ideal of $S$. Similarly $S^{p} B^{\prime} S^{q}$ is an $(p, q)$-lateral ideal and $(S S)^{n-1} B^{\prime}$ is an $n$-left ideal of $S$. Since $R, M$ and $L$ are minimal $m$-right ideal, minimal $(p, q)$-lateral ideal and minimal $n$-left ideal of $S$ respectively, therefore $B^{\prime}(S S)^{m-1}=R, S^{p} B^{\prime} S^{q}=$ $M$ and $(S S)^{n-1} B^{\prime}=L$. Thus $B=R M L=B^{\prime}(S S)^{m-1} S^{p} B^{\prime} S^{q}(S S)^{n-1} B^{\prime} \subseteq B^{\prime}$. Hence $B=B^{\prime}$. Consequently, $B$ is minimal $(m,(p, q), n)$-bi-ideal of $S$.

Definition 5.2. Let $S$ be a ternary semigroup. Then $S$ is called a bi-simple ternary semigroup if $S$ is the unique $(m,(p, q), n)$-bi-ideal of $S$.

Theorem 5.2. Let $S$ be a ternary semigroup and $B$ be an $(m,(p, q), n)$-bi-ideal of $S$. Then $B$ is a minimal $(m,(p, q), n)$-bi-ideal of $S$ if and if $B$ is a bi-simple ternary semigroup.

Proof. Suppose $B$ is a minimal $(m,(p, q), n)$-bi-ideal of $S$. Let $C$ be an $(m,(p, q), n)$ bi-ideal of $B$. Then $C(B B)^{m-1} B^{p} C B^{q}(B B)^{n-1} C \subseteq C \subseteq B$. By Proposition 4.9, $B C C$ is an $(m,(p, q), n)$-bi-ideal of $S$. Therefore

$(B C C)(S S)^{m-1} S^{p}(B C C) S^{q}(S S)^{n-1} B C C \subseteq B C C \subseteq B B B \subseteq B$. Since $B$ is minimal, therefore $B C C=B$. It is easy to show that $C(B B)^{m-1} B^{p} C B^{q}(B B)^{n-1} C$ is an $(m,(p, q), n)$-bi-ideal of $S$.

Since $B$ is minimal, therefore $C(B B)^{m-1} B^{p} C B^{q}(B B)^{n-1} C=B$. This implies $B=C(B B)^{m-1} B^{p} C B^{q}(B B)^{n-1} C \subseteq C$. Hence $C=B$. Consequently, $B$ is a bisimple ternary semigroup.

Conversely, suppose $B$ is a bi-simple ternary semigroup. Let $C$ be an $(m,(p, q), n)$ bi-ideal of $S$ such that $C \subseteq B$. Then

$C(B B)^{m-1} B^{p} C B^{q}(B \bar{B})^{n-1} C \subseteq C(S S)^{m-1} S^{p} C S^{q}(S S)^{n-1} C \subseteq C$

which implies that $C$ is an $(m,(p, q), n)$-bi-ideal of $B$. Since $B$ is bi-simple ternary semigroup, therefore $C=B$. Hence $B$ is minimal.

\section{References}

[1] F. M. Sioson, Ideal theory in ternary semigroups, Math. Japon.,10 (1965), 6384.

[2] O. Steinfeld, Quasi-ideals in rings and semigroups, Akademiai Kiado, Budapest (1978).

[3] R. Chinram, A note on $(m, n)$ Quasi-ideals in semigroups, International Journal of Pure and Applied Mathematics, 49 (1), (2008), 45-50.

[4] R. Chinram, Generalised quasi-ideals of semigroups, KKU Sci. J., 37 (2), (2009), 213-210. 
[5] R. A. Good and D. R. Hughes: Associated groups for a semigroup, Bull. Amer. Math. Soc. 58 (1952), 624 625:

[6] S. Kar, On quasi-ideal and bi-ideals in ternary semiring, Int. J. Math. ES Math. Sci., 18, (2005), 3015-3023.

[7] V.N. Dixit and S. Dewan, A note on quasi and bi-ideals in ternary semigroups, Int. J. Math. E Math. Sci. 18 (3), (1995), 501-508.

DOI: $10.7862 /$ rf.2014.3

\section{Manish Kant Dubey}

email: kantmanish@yahoo.com

SAG, DRDO, Metcalf House,

Delhi 110054, India.

Rahatgi Anuradha - corresponding author

email: anuvikaspuri@yahoo.co.in

Department of Mathematics,

University of Delhi,

Delhi 110007, India.

Received 5.02.2013, Accepted 25.10.2013 